water, fuel, and equipment, made it a herculean task. It was a blessing that the typhus season was well advanced by this time. The weather was hot, and the prisoners wore the minimum of clothing. As organized prisoner-of-war cages were established, and satisfactory arrangements were made, the danger passed.

\section{Relapsing Fever}

The presence of this disease in North Africa has a bearing on this discussion. Relapsing fever is endemic in Algeria, and during 1943 twelve British troops were affected. The disease increased to epidemic form at the end of 1944 and early in 1945. It spread quickly from Algeria to Tunisia, where 1,746 cases occurred during the last two weeks of February. In January, 1945, there were 6,000, and in February 7,400 cases, including 80 weekly in a civil prison. It was reported that the epidemic was assuming enormous proportions in the Saharan towns and those of the Tel plateau. This disease is spread in epidemic form by lice; it is thus evident that the native infestation rate was still very great. One would therefore have expected the incidence of typhus to remain at a high level. The fact that a large number of the Arabs had already had typhus is not sufficient to account for the dramatic fall in morbidity in 1943, 1944, and 1945 in a heavily infested and economically depressed people. Vaccination was therefore effective despite the fact that infestation remained.

\section{Reasons for the Low Incidence of Typhus among Allied Troops}

Of first importance was the fall in the number of Arab cases in 1943, when the anticipated increase did not occur. This was probably the result of the mass vaccination scheme of the previous two years. Vaccination was continued in 1943, when notices appeared in the towns and in the Press stating that it was compulsory. All persons between 10 and 70 years were required to present themselves for inoculation; fines ranging from 100 to 1,000 francs were payable in default.

The fear of typhus, stimulated by intensive propaganda, made a great impression on the troops, and was a potent reason for our escape. The military police, despite their numerous other commitments, gave great help in enforcing orders and in ensuring that " out of bounds" notices were not ignored. Bath discipline was good. A.L.63 powder, although often used carelessly and irregularly, was extremely effective. The wide dispersion of units and the careful selection of camps probably helped also. Inoculation was not a factor of any importance, for until the late spring and summer the number protected was negligible.

(Part II will appear in next week's issue.)

\section{DUODENAL ULCERATION : A GASTROSCOPIC STUDY OF THE GASTRIC MUCOSA AND ITS SURGICAL SIGNIFICANCE}

HARRY FREEMAN, F.R.C.S.

Surgical Registrar, National Temperance Hospital; Surgeon, E.M.S.; Assoc. Lecturer on Surgery, North-East London Postgraduate College

The problems associated with the surgical treatment of duodenal ulceration and of some of the complications which follow in its wake in a very large proportion of cases have for many years occupied the mind of the profession, yet it seems that little universal progress has been made in the past quarter of a century in the treatment of a disease which still produces much physical and mental suffering in the individual and much economic loss to the community at large. With these problems in mind, I have made an intensive gastrosçopic study of the mucosa of the stomach in the presence of duodenal ulceration, as well as a study of the mucosa in cases following operations for this malady, with a view to eliciting the relationship of the anatomy and physiology of the morbid mucosa and its transmutation as a result of surgical treatment, and its bearing upon the subsequent condition of the patient.

A close study of the aetiological factors said to be associated with duodenal ulceration reveals that a definite relationship exists between this pathology and a deranged physiology of the stomach and, indeed, that of the greater part of the alimentary tract. It would therefore be as well to give consideration to the individual who presents himself with established ulceration or, on the other hand, merely with symptoms suggesting the outlines of the image, which, as time goes on, gradually evolves itself into the completed picture of a subject with duodenal ulceration. $\mathrm{He}$ is an extremely sensitive and hyper-irritable type, in a constant state of nervous tension and emotional instability; vásomotor disturbances are present and, above all, he is stomach-conscious. These nervous influences play a very important part by virtue of the fact that they are referred to his alimentary tract and can be readily demonstrated by the radiologist during an $x$-ray examination after a barium meal. They further manifest themselves in certain spastic phenomena, of which spasm of the pylorus and duodenum and of the descending colon are typical examples. So very often little importance is attached to these phenomena at the time of the examination or, indeed, to any subsequent report of these radiological findings or their possible interpretation. When spasm occurs in a vascular structure a temporary impairment of the blood supply to the part concerned results, with local loss of viability. These changes commonly occur in the duodenum, and the local loss of viability produces a condition in which the mucosa becomes vulnerable to injury by contact with irritant or insufficiently digested food; if in addition local necrosis occurs it is not difficult to visualize the possibility of ulceration in the presence of a high acid-pepsin content of the gastric juice. The duodenum houses a mechanism which is stimulated by the presence of food into liberating the hormone enterogastrone, which itself controls gastric secretion and peristalsis. When spasm and local loss of viability occur this mechanism fails, and the normal gastric function is now replaced by hypermotility and rapid emptying of insufficiently digested food, which may damage the vulnerable duodenal mucosa; such injury is aggravated by the high acid secretion which is a constant factor in the type of individual described.

The surgeon is frequently called upon to deal with a perforation of or haemorrhage from a peptic ulcer, but a precise history of gastric symptoms is very often lacking in these examples of "silent ulcer"; later inquiry into the health and habits of these patients reveals that the various nervous phenomena did however exist, and even at an early age a testmeal examination would show a marked increase in the amount and acid content of the gastric juice.

\section{Normal Gastric Mucosa}

As seen through the gastroscope the gastric mucosa consists of a thick transparent smooth orange-red lining thrown into many folds throughout the greater part of the stomach. These folds are tortuous in some areas and resemble the convolution: of the cerebral cortex. The folds can be straightened out or made to disappear altogether when the stomach is distended with air, and this is an important point in differentiating the normal from the hyperplastic type of mucosa, in which the folds in

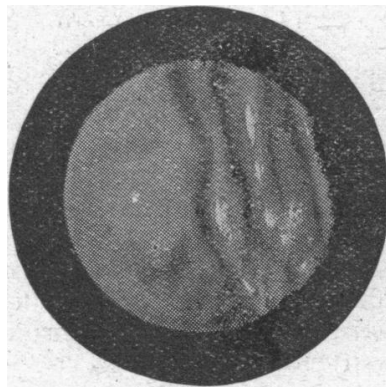

Fig. 1.-Normal mucosa on anterior wall (left of picture) and greater curvature.

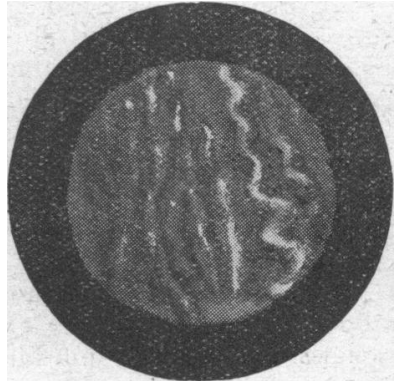

FIG. 2.-Normal mucosa on posterior wall and greater curvature with little inflation. certain areas remain tortuous even with full inflation. The smoothest part of the mucosa is found along the lesser curvature of the stomach, but occasionally one or two very thin parallel folds may be seen here. Both the greater curvature and the posterior wall of the stomach present a network of many folds, parallel and slightly tortuous in the former situation, but much 
more tortuous and thicker on the posterior wall (Figs. 1 and 2). A few thin parallel folds are present in the pyloric antrum, but these readily disappear with very little inflation and are not often observed. The surface of the mucosa is very smooth and glistening in all areas of the stomach; many high lights are sprinkled over the gastroscopic picture, and these are due to the diffused glow of the lamp over the moist surface. When secretion is excessive the number of high lights is correspondingly increased; if gastritis is present and some of the ducts become blocked, secretion into the cavity of the stomach is diminished and the number of high lights seen is very small, or they may be absent altogether.

\section{Gastric Mucosa in the Presence of Duodenal Ulcer}

In a series of 50 gastroscopic examinations of cases of duodenal ulceration $I$ found that in $76 \%$ the mucosa was of the hyperplastic type; the mucosa of the remainder varied a little in the development of the folds, but was considered to be within normal limits. This hyperplasia manifests itself in the formation of very many crowded, thickened, and extremely tortuous folds which cannot be straightened out even after full inflation of the stomach, and which certainly cannot be made to disappear. These crowded folds are most marked upon the posterior wall of the stomach; they develop along the greater curvature and may replace the straight folds normally present on the anterior wall. There is thus found a more or less generalized hyperplasia or hypertrophy of the gastric mucosa, with an increased complement of glandular elements which is capable of producing an excessive secretion of a hyper-acid juice.

\section{Gastric Mucosa in Non-obstructive Cases}

In addition to generalized hyperplasia gastritis may be present, and this manifests itself by areas of oedematous folds and scattered patches of viscid adherent greyish-yellow secretion, which occupies chiefly the valleys between these folds; high lights are markedly diminished over these areas of superficial gastritis, and the mucosa is vulnerable and may readily bleed during the examination. Gastritis is not necessarily an accompaniment of duodenal ulceration, but, when present, disappears with adequate medical treatment although the ulcer itself may still remain. When an ulcer has healed under a medical regime the hyperplastic nature of the mucosa does not change. Sometimes a gastric ulcer may also be present in addition to the duodenal ulcer, and I have observed three such cases in the series. The incidence of this phenomenon varies, and has no relation to the mucosa of the stomach; Carey and Ylvisaker (1942) have reported as many as 9 out of a series of 70 cases of duodenal ulceration in which gastric ulcer was also present.

\section{Mucosa in Obstructive Cases}

It is not sufficiently appreciated that obstructive symptoms following upon ulceration may be entirely due to local oedema and spasm of the pylorus. First, this is borne out by the fact that cases in which the radiologist reports a delayed emptying of the stomach will improve with adequate medical treatment and a little patience if the cause is essentially one of oedema and spasm, and it is always worth while repeating the $x$-ray examination two or three times after intervals of three weeks. Secondly, a large number of these cases are operated upon following the report of obstruction, and a gastro-enterostomy performed. When these patients are subsequently seen because of a recurrence of symptoms, and a fresh $x$-ray examination is carried out, it may surprise the surgeon when the radiologist reports that "a large portion of the meal passes through the pylorus." Obviously, then, these were cases where the obstruction was non-fibrous and purely transient.

The condition of the mucosa in these obstructive cases varies considerably; viscid adherent secretion and a dulling of or granular change in the surface of the mucosa constantly take place over large areas, but these mucosal phenomena disappear when the obstruction is relieved. If, however, obstruction has periodically occurred, or if the obstruction is of the fibrous variety and progressive, the changes seen in the mucosa are very much more marked: not only is there an increase of the pathological secretion but the folds are very oedematous and eventually become broken up into raised nodular segments which resemble pavement or cobble stones closely packed together.
These changes occur in the valleys between the folds as well as on the folds themselves. This condition is sometimes known by the name of "chronic hypertrophic gastritis," and must not be confused with simple hyperplasia of the mucosa. Occasionally the pyloric antrum becomes the seat of these changes, but only after the onset of true fibrous obstruction.

\section{Fifty Cases of Duodenal Ulcer}

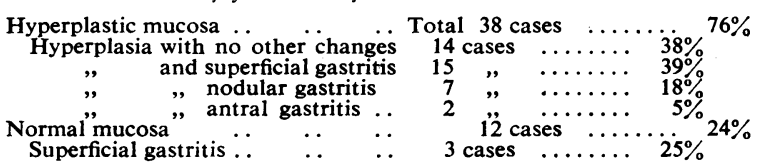

\section{Post-operative Recurrence of Symptoms}

It is not my intention to enumerate the many causes of recurrence of symptoms after operation for duodenal ulceration. Patients often present themselves with symptoms similar to those for which they were operated upon. These symptoms may be due to inflammatory changes which were present in the gastric mucosa before operation, and which persist after operation. It may be well worth while to gastroscope the patient before any operation is contemplated, in order to visualize the gastric mucosa and its condition. With the failure of the short-circuit operation in many instances-for this had become a disease in itself rather than an intended cure-more radical measures were resorted to. Although advocates of gastroduodenectomy and partial gastrectomy have shown individually results which should be very convincing, yet it is a fact that these formidable procedures still have a high mortality in some hands, and even a great degree of morbidity in others. Stomal ulceration may occur after partial gastrectomy (Fig. 3), and I have observed such cases ; doubtless more

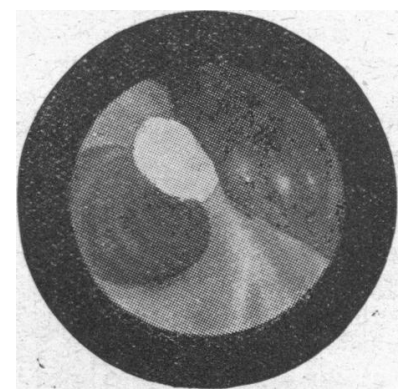

Fig. 3.-Stomal uicer 18 months after partial gastrectomy.

cases will be recorded as gastroscopic examinations are increased. Inflammatory changes in the mucosa of the gastric stump must also be included in this category, so that this operation is not altogether a solution for a very important problem in surgery. In addition, the creation of a very grossly distorted anatomy and a grossly deranged physiological function of the stomach does not seem rational in the quest for a cure of a condition the aetiology of which is still unknown. The initial success of partial gastrectomy may be hailed by both patient and surgeon, but the morbidity is not quite appreciated because with a recurrence of symptoms a large number of these patients change their hospital. Even at the best the patient fails to regain weight or to admit of being comfortable; he remains' stomach-conscious.

To ensure success of any medical regime in the treatment of duodenal ulceration it is essential that the amount of gastric juice and the degree of acidity be considerably reduced; whichever method achieves its object in healing the ulcer, the lowered acid content of the gastric juice is but a passing phase. When surgical measures are indicated it is desirable not only to obtain a physiological reduction in the activity of the stomach but to ensure that this reduction is permanent or at least prolonged, because very real risks of complications ensue on any surgical procedure if the original high acidity remains. The high-acidsecreting hyperplastic type of mucosa is very liable to stomal ulceration after the operation of gastro-enterostomy, and indeed after any surgical procedure if the high acidity is allowed to remain. Of those cases of stomal ulcer observed by me in the course of gastroscopic examinations, $65 \%$ occurred in the 
presence of hyperplasia of the gastric mucosa. This operation alone should clearly be avoided when the mucosa of the stomach is of this type. Bearing this all-important problem in mind, it may be more rational to adopt a simpler and apparently surer method of attaining what the more formidable methods have failed to do, and in this respect we have seen some excellent results which have been obtained by Somervell (1942), who advocates ligature of a large proportion of the arteries which supply the stomach. The hypersecretion and acidity are immediately reduced, and remain so for a very long time.

In order to evaluate the results of ligature, I have observed the changes in the mucosa of the stomach in which I myself carried out this operation. It was seen that the mucosa in simple hyperplasia had undergone a marked transmutation from the hyperplastic state to one which could be considered as being well within normal limits. These cases were gastroscoped before (Fig. 4) and six months after (Fig. 5) gastric arterial ligature

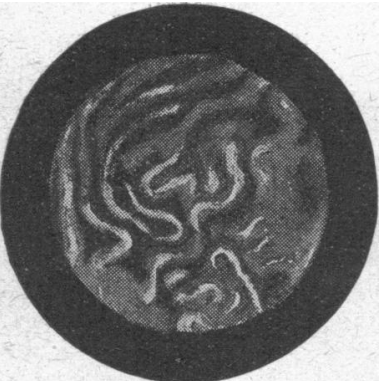

Fig. 4.-Case of duodenal ulceration: hyperplastic mucosa with crowded folds.

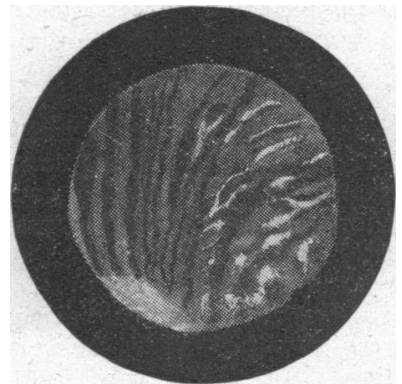

Fig. 5.- Same case as in Fig. 4 six months after arterial ligature. Note regression of folds. had been performed; the folds were now far less crowded and tortuous, and inflation straightened many of them. In the presence of fibrous obstruction, posterior gastro-enterostomy was performed in addition to ligature, but where nodular changes in the mucosa had occurred these remained unchanged after an interval of six months. It is possible that earlier treatment would have prevented these nodular changes from developing.

Although these conclusions have been drawn from a limited number of cases, it is possible to correlate the reduction of gastric acidity with the changes in the nature of the mucosa following upon ligature. This regression must be part of an inherent power peculiar to the mucosa, in which reverse changes -namely, regeneration under certain conditions - may also take

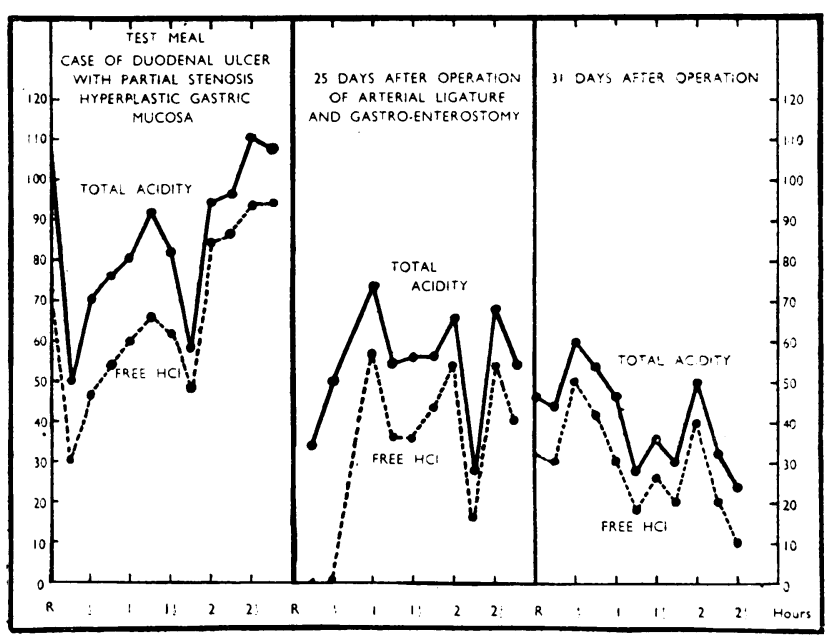

Chart of a case of duodenal ulcer.

place; the thinned atrophic mucosa associated with pernicious anaemia will regenerate and regain its thickness with suitable liver therapy, and the reappearance of folds will produce a picture which is difficult to distinguish from the normal.

It is thus reasonable to conclude that there is sufficient justification to look upon these more conservative surgical measures in the treatment of duodenal ulceration as heralding a tremen- dous field of opportunity for further work in this direction. The clinical and physiological results, as well as the gastroscopic findings following upon arterial ligature alone, or in conjunction with gastro-enterostomy (see accompanying Chart of a case) if fibrous obstruction is present, are such that even the most sceptical would agree on the value of, and the desirability for pursuing, measures less drastic than partial gastrectomy if these measures attain their object in reducing the high acid content of the gastric secretion without a gross distortion of the anatomy and physiology of the stomach.

REFERENCES

Carey, J. B., and Ylvisaker, R. S. (1942). Lancet, 62, 394. Somervell, T. H. (1942). Brit. J. Surg., 30, 113.

\section{THEORY AND NOMENCLATURE OF THE Rh, TYPES, SUBTYPES, AND GENOTYPES

\author{
BY
}

\section{ALEXANDER S. WIENER, M.D., F.A.C.P.}

The $\mathrm{Rh}$ blood types have been the subject of many communications to the Journal. The purpose of this article is to correct several statements which have appeared in some of these reports, in order to prevent widespread confusion in an already highly complicated subject.

In a recent letter to Nature ${ }^{1}$ Fisher and Race state: "The notation has been frequently changed, and we feel that only a notation which designates unambiguously the antibodies, the genes or gene-complexes, and the antigens with which these antibodies react, can avoid widespread confusion." With the latter part of this statement I agree whole-heartedly, but would add that the notations should be made as simple as possible to favour their fluent use, both orally and in print, by medical men and geneticists, in order to further the work in the field. This has been my aim ever since my first work $^{23}$ on the $R h$ types was done in 1940-2, at which time only the anti- $R h_{0}$ and anti-R $\mathbf{h}^{\prime}$ agglutinins were available. Changes were made only when advances in knowledge, such as the discovery of the agglutinin anti- $\mathrm{Rh}^{\prime \prime}$ and the $\mathrm{Hr}$ agglutinins, made slight revisions and additions to the nomenclature necessary. As explained by $\mathrm{me}^{4}$ in a historical article presented before the New York Academy of Sciences, and also by Taylor and Race, ${ }^{5}$ these new findings did not change our previous results but merely added to them, and in revising the nomenclature as many of the old names as possible were always retained. The new findings required the creation of some new names, but at all times the principle of keeping the designations as simple as possible and unambiguous guided my actions. Some confusion has been created by the attempt of other workers ${ }^{6}$ to introduce numbered nomenclatures which fail to take into account the established genetic and serologic knowledge of the subject. Considering only the four agglutinins-anti- $\mathrm{Rh}^{\prime}$, anti-

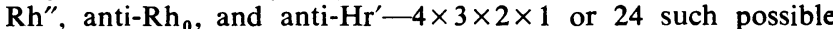
numberings can be devised, so if the use of numbers were encouraged a far worse confusion would result than the MossJansky jumble. Luckily, such numbered systems have found favour only in the laboratories of their originators, and so have caused little or no harm to date.

The letter by Fisher and Race continues: "Six of the gene designations here adopted are due to Wiener, but for the antibodies his notation seems arbitrary. While Cappell's names, such as anti-C, anti-D, anti-E, and anti-c, are unambiguous, Wiener's do not seem satisfactory, since, for example, the $85 \%$ reacting serum is called anti- $R h_{0}$ whereas besides $R h_{0}$ it reacts with the genes $R h_{1}, R h_{2}$, and $R h_{z} . "$ As can be seen from Table I.

TABLE I.-Scheme of the Eight Rh Blood Types

\begin{tabular}{|c|c|c|c|c|c|c|c|}
\hline \multicolumn{4}{|c|}{$\begin{array}{l}\text { Clinically Rh-negative Individuals } \\
(15 \%)\end{array}$} & \multicolumn{4}{|c|}{$\begin{array}{c}\text { Clinically Rh-positive Individuals } \\
(85 \%)\end{array}$} \\
\hline \multirow{2}{*}{$\begin{array}{l}\text { Designations } \\
\text { of Types }\end{array}$} & \multicolumn{3}{|c|}{$\begin{array}{c}\text { Reactions with } \\
\text { Antisera }\end{array}$} & \multirow{2}{*}{$\begin{array}{l}\text { Designations } \\
\text { of Types }\end{array}$} & \multicolumn{3}{|c|}{$\begin{array}{l}\text { Reactions with } \\
\text { Antisera }\end{array}$} \\
\hline & $\mathbf{R h}^{\prime}$ & $\mathbf{R h}^{\prime \prime}$ & $\mathrm{Rh}_{0}$ & & $\mathbf{R h}^{\prime}$ & $\mathbf{R h}^{\prime \prime}$ & $\mathbf{R h}_{\mathrm{n}}$ \\
\hline $\begin{array}{c}\text { rh } \\
\mathbf{R h}^{\prime} \\
\mathbf{R h}^{\prime \prime} \\
\mathbf{R h}^{\prime} \mathbf{R h}^{\prime \prime}\end{array}$ & $\begin{array}{l}\text { Neg. } \\
\text { Pos. } \\
\text { Neg. } \\
\text { Pos. }\end{array}$ & $\begin{array}{l}\text { Neg. } \\
\text { Neg. } \\
\text { Pos. } \\
\text { Pos. }\end{array}$ & $\begin{array}{l}\text { Neg. } \\
\text { Neg. } \\
\text { Neg. } \\
\text { Neg. }\end{array}$ & $\begin{array}{c}\mathrm{rh}_{0} \\
\mathrm{Rh}_{1}\left(\mathrm{Rh}_{0}^{\prime}\right) \\
\mathrm{Rh}_{2}\left(\mathrm{Rh}_{0}^{\prime \prime}\right) \\
\mathrm{R} \mathrm{h}_{1} \mathbf{R h _ { 2 }}\end{array}$ & $\begin{array}{l}\text { Neg. } \\
\text { Pos. } \\
\text { Neg. } \\
\text { Pos. }\end{array}$ & $\begin{array}{l}\text { Neg. } \\
\text { Neg. } \\
\text { Pos. } \\
\text { Pos. }\end{array}$ & $\begin{array}{l}\text { Pos. } \\
\text { Pos. } \\
\text { Pos. } \\
\text { Pos. }\end{array}$ \\
\hline
\end{tabular}

